\title{
Lactobacillus rhamnosus Granules Dose-Dependently Balance Intestinal Microbiome Disorders and Ameliorate Chronic Alcohol-Induced Liver Injury
}

\author{
Zelin Gu, ${ }^{1}$ Yanfeng Wu, ${ }^{1}$ Yu Wang, ${ }^{1}$ Haiyue Sun, ${ }^{1}$ Ying You, ${ }^{1}$ Chunhong Piao, ${ }^{1,2}$ \\ Junmei Liu, ${ }^{1,2}$ and Yuhua Wang ${ }^{1-3}$ \\ ${ }^{1}$ College of Food Science and Engineering, Jilin Agricultural University, Changchun, China. \\ ${ }^{2}$ National Processing Laboratory for Soybean Industry and Technology, Jilin Agricultural University, Changchun, China. \\ ${ }^{3}$ National Engineering Laboratory for Wheat and Corn Deep Processing, Jilin Agricultural University, Changchun, China.
}

\begin{abstract}
As the functions of Lactobacilli become better understood, there are increasing numbers of applications for Lactobacillus products. Previously, we have demonstrated that Lactobacillus rhamnosus GG (LGG) can prevent alcoholic liver injury. LGG granules were produced by fluid bed granulation with a media composed of starch, skimmed milk powder, whey powder, microcrystalline cellulose and maltose, and LGG fermented liquid that comprised 30-50\% of the total weight. We found LGG granules dose-dependently protected against chronic alcoholic liver disease. When alcohol was consumed for 8 weeks with LGG treatment during the last 2 weeks, we demonstrated that the dose dependence of LGG granules can improve alcoholinduced liver injury through decreasing the levels of lipopolysaccharide and tumor necrosis factor- $\alpha$ in serum and prevent liver steatosis by suppressing triglyceride, free fatty acid, and malondialdehyde production in liver. Alcohol feeding caused a decline in the number of both Lactobacillus and Bifidobacterium, with a proportional increase in the number of Clostridium perfringens in ileum, and expansion of the Gram-negative bacteria Proteobacteria, Campylobacterales, and Helicobacter in cecum. However, LGG granule treatment restored the content of these microorganisms. In conclusion, LGG granule supplementation can improve the intestinal microbiota, reduce the number of gram-negative bacteria, and ameliorate alcoholic liver injury.
\end{abstract}

\section{KEYWORDS: • alcohol • intestinal flora • Lactobacillus rhamnosus GG granules • liver injury}

\section{INTRODUCTION}

A LCOHOLIC LIVER INJURY is caused by excessive alcohol consumption. Long-term drinking is one of the major causes of chronic liver injury that includes diseases such as simple fatty liver and serious liver diseases such as liver steatosis, liver cirrhosis, and liver cancer. ${ }^{1-3}$ Some mild alcoholic liver injury could be reversed if drinking is discontinued. However, the more serious liver injury, such as cirrhosis, is irreversible. ${ }^{4}$ At present, no drug is approved by Food and Drug Administration for the treatment of alcoholic liver disease (ALD). With the discovery of a close relationship between intestinal microflora and ALD, the potential use of probiotics in ALD is receiving increasing investigative and clinical attention.

According to the Food and Agriculture Organization of the United Nations/World Health Organization (FAO/WHO) definition, probiotics are defined as "living microorganisms that, when administered in adequate amounts, confer a health benefit on the host." ${ }^{5,6}$ Probiotics and pro-

Manuscript received 7 November 2018. Revision accepted 11 September 2019.

Address correspondence to: Yuhua Wang, PhD, College of Food Science and Engineering, Jilin Agricultural University, Xin-Cheng Street, Changchun 130118, China,E-mail: yuhua-ww@163.com biotic metabolites have the potential to directly or indirectly adjust the composition of the host intestinal microbiota ${ }^{7}$ and activate the endogenous microbial community and regulate the immune system. ${ }^{8-11}$ Currently, the most often used probiotics are lactic acid bacteria (LAB), Bifidobacteria, ${ }^{7}$ Propionibacteria, yeasts (Saccharomyces boulardii), and the Gram-negative Escherichia coli strain Nissle 1917. ${ }^{12}$ Lactobacilli are the main component of LAB, which are frequently used probiotics. Various species and strains of Lactobacilli have been used to treat ALD in animals and humans, including Lactobacillus rhamnosus GG (LGG) ${ }^{13-15}$ Lactobacillus acidophilus, ${ }^{16}$ Lactobacillus helveticus, ${ }^{17}$ VSL\#3, ${ }^{18,19}$ Lactobacillus casei, ${ }^{20}$ heat-killed Lactobacillus brevis $\mathrm{SBC} 8803,{ }^{21}$ and Lactobacillus plantarum 8PA3. ${ }^{6}$

Among those, LGG is the most frequently used strain. LGG is a short Gram-positive heterofermentative facultative anaerobe that was isolated in 1983. Previous study showed that LGG can effectively treat alcoholic liver injury. LGG can improve alcoholic fatty liver enzyme levels in patients with hepatitis ${ }^{6}$ and alcoholic liver injury in experimental animals. ${ }^{13,14}$ Our previous studies also showed LGG and LGG culture supernatant (LGG-s) can enhance intestinal barrier function, modulate mucosal immune system, and improve hepatic lipid metabolism to relieve alcohol-induced liver injury by regulating intestinal and hepatic signal factor 
expression. ${ }^{13,14,22}$ Our results found that 8 -week chronic alcohol feeding decreased the number of Bacteroides and Firmicutes, increased the number of Proteobacteria and Actinobacteria in the mice feces. Alcohol induced the expansion of Corynebacterium and Alcaligenes, and the proliferation of Alcaligenes increased the intestinal $\mathrm{pH}$ and led to pathogenic bacteria overgrowth, while the LGG administration suppressed the overgrowth of Alcaligenes and Corynebacterium in the mice feces. ${ }^{14}$

In the present study, we discovered that the dosedependent effects of LGG granules ameliorated chronic alcohol-induced liver injury in a C57BL/6N mouse model and resulted in a beneficial modification of gut microbiota including ileum and cecum.

\section{MATERIALS AND METHODS}

\section{Probiotic strains and LGG granules}

LGG was purchased from the American Type Culture Collection (accession 53103; ATCC, Rockville, MD) and was cultured in Lactobacillus De Man, Rogosa, and Sharpe broth (Difco MRS Broth; BD, Sparks, MD, USA) at $37^{\circ} \mathrm{C}$ in accordance with ATCC guidelines, which was prepared as described previously. ${ }^{22}$ LGG was cultured after 3 generation cultures in the MRS broth. LGG granules were produced by fluidized bed granulator, which combined LGG fermented broth that is 30$50 \%$ of the total weight and starch, skimmed milk powder, whey powder, microcrystalline cellulose and maltose (the ratio was $4: 1: 1: 1: 1)$. Length of LGG granules was 1-3 mm.

\section{Animal feeding}

The experiments were performed on male C57BL/6N mice aged 8 weeks that were provided by the Experimental Animal Center, Bethune Medical College, Jilin University (Jilin, China; Certificate No.: SCXK (Ji) 2016-0001). The mice were cared according to the protocols of the Institutional Animal Care and Use Committee of the Jilin University. Animals were housed in an institutional animal care facility with $12 \mathrm{~h}$ light and dark cycles. All mice had free access to regular laboratory chow and water until the start of experiments. During the experiments, they were fed a liquid diet as described below.

C57BL/6N male mice were fed in College of Public Health in Jilin University. The mice were pair-fed liquid diet (Lieber DeCarli) (contains $17 \%$ of energy as protein, $40 \%$ as corn oil, $7.5 \%$ as carbohydrate) and $35.5 \%$ as either alcohol (alcohol-fed, AF) or isocaloric maltose dextrin (pair-fed, $\mathrm{PF}$ ) in the following groups: $\mathrm{PF}, \mathrm{AF}, \mathrm{AF}+\mathrm{LGG}$, and an overall control group of normal chow (13\% of energy from fat, Normal chow), ${ }^{13}$ and in AF + LGG group there were three patterns about the use level of LGG granules at deferent dose of $0.05,0.1,1.0 \mathrm{~g} /($ day $\cdot$ mouse $)$. Alcohol consumption continued for 8 weeks and LGG granules were fed at the last 2 weeks. The mice were fed different doses of LGG granules (The level was low, middle, and high.) mixed with a high fat diet. Five treatment groups were studied: (1) PF $(n=7)$, (2) AF $(n=12),(3) \mathrm{AF}+\mathrm{LGGL}$ (low dose) $(n=15),(4) \mathrm{AF}+\mathrm{LGGM}$ (middle dose) $(n=15)$, and $(5)$ $\mathrm{AF}+\mathrm{LGGH}$ (high dose) $(n=15)$.

\section{Liver histopathology}

Formalin-fixed, paraffin-embedded tissue, accumulation of hepatic fat was determined by hematoxylin and eosin (H\&E) staining and then studied by light microscopy, as previously described. ${ }^{13}$

\section{Serum biochemical assay}

Serum was obtained by centrifuging the blood at $3000 \mathrm{~g}$ for $10 \mathrm{~min}$ at $4^{\circ} \mathrm{C}$. Serum alanine aminotransferase (ALT) and aspartate aminotransferase (AST) were determined using ALT and AST Infinity Assay Kits (Thermo Scientific), respectively. Lipopolysaccharide (LPS) in serum was determined using analytical reagent kit (Nanjing Jiancheng Bioengineering Institute, China). Serum levels of tumor necrosis factor- $\alpha$ (TNF- $\alpha)$ were measured using TNF- $\alpha$ Mouse ELISA Kit (R\&D Systems, Minneapolis, MN, USA).

\section{Liver assays}

A total of $0.1 \mathrm{~g}$ liver tissue was homogenized with $0.9 \mathrm{~mL}$ of $0.9 \%$ saline using a homogenizer (Biospec Products, Racine, WI, USA). Liver supernatant was obtained by centrifuging liver homogenate at $1800 \mathrm{~g}$ for $10 \mathrm{~min}$ at $4^{\circ} \mathrm{C}$. Triglyceride (TG), free fatty acids (FFAs), and malondialdehyde (MDA) were determined using analytical reagent kits (Nanjing Jiancheng Bioengineering Institute, China).

\section{Traditional culture of intestinal flora}

A total of $0.1 \mathrm{~g}$ feces samples were homogenized with $0.9 \mathrm{~mL}$ of $0.9 \%$ saline using a homogenizer (Biospec Products). A feces sample supernatant was obtained by centrifuging at $1800 \mathrm{~g}$ for $10 \mathrm{~min}$ at $4^{\circ} \mathrm{C}$. The supernatant was diluted to $10^{1}-10^{8}$ times, and then the suitable dilutions were coated on a culture plate filled with a solid medium. Lactobacillus, Bifidobacterium, and Clostridium perfringens were cultured respectively and repeated three times for statistical analysis after counting.

\section{$16 S$ rRNA gene sequencing analysis}

The cecum of 7 mice in the PF group, 10 and 15 mice in AF group and AF + LGG group (middle dose) were used for the metagenomic analysis. The contents of the cecum were isolated to extract bacterial genomic DNA using TIANamp Stool DNA Kit (cat. no. DP328; Spin Column) according to the manufacturer's recommendations. Fragments including V4 16S rDNA hypervariable regions were amplified by polymerase chain reaction (PCR) using the forward primer $520 \mathrm{~F}$ and $802 \mathrm{R} .{ }^{23}$ The amplification conditions were $94^{\circ} \mathrm{C}$ for $5 \mathrm{~min}$ (initial denaturation), followed by 25 cycles of $94^{\circ} \mathrm{C}$ for $4 \mathrm{~min}, 50^{\circ} \mathrm{C}$ for $30 \mathrm{sec}$ (annealing) and $72^{\circ} \mathrm{C}$ for $30 \mathrm{sec}$ (extension), and final extension at $72^{\circ} \mathrm{C}$ for $5 \mathrm{~min}$. The PCR products were separated on $2 \%$ agarose gel, and 
that was purified using AxyPrep Gel Extraction Kit (Axygen, Hangzhou, China), which were sequenced using Illumina MiSeq platform (HiSeq 2000).

Then, operational taxonomic unit (OTU) and taxonomic classification sequences were used to analyze the data. The taxonomic classification of the sequence reads were discriminated by the Ribosomal Database Project (RDP). ${ }^{24}$ Classification of sequences from phylum was performed down to the genus level, the phylum from $97 \%$ similarity threshold, the family of $90 \%$ similarity threshold, and the genus of $80 \%$ similarity threshold. The table of OTU was analyzed by the Mothur ${ }^{25}$ and Qiime ${ }^{26}$ software packages. The alpha diversity and the beta diversity were determined by distance-based classification and phylogeny from the filtered sequences.

\section{Statistical analysis}

All values were expressed as mean standard error (mean \pm standard error). Analysis of variance with Tukey's multiple comparison test was performed to determine sta- tistical significance using GraphPad Prism 5.0. values of $P<.05$ and $P<.01$ were considered statistically significant.

\section{RESULTS}

\section{Effect of LGG granules supplementation on chronic alcohol-induced liver steatosis}

The effect of LGG granule treatment on chronic alcoholinduced liver fat accumulation was compared by histological analysis and biochemical analysis. The accumulation of liver lipid was significantly increased in the AF group, LGG treatment can improve chronic alcohol-induced liver steatosis, which was evaluated by H\&E staining (Fig. 1A). The liver injury was evaluated by detecting the serum ALT and AST. In AF group, serum ALT (Fig. 1B) and AST (Fig. 1C) levels were significantly increased compared with PF group and significantly attenuated in the LGG granule treatment group. Furthermore, the higher concentration of TG reflects the more serious liver steatosis due to oxidative stress and lipid metabolism disorders. We found the hepatic TG level

\section{A}
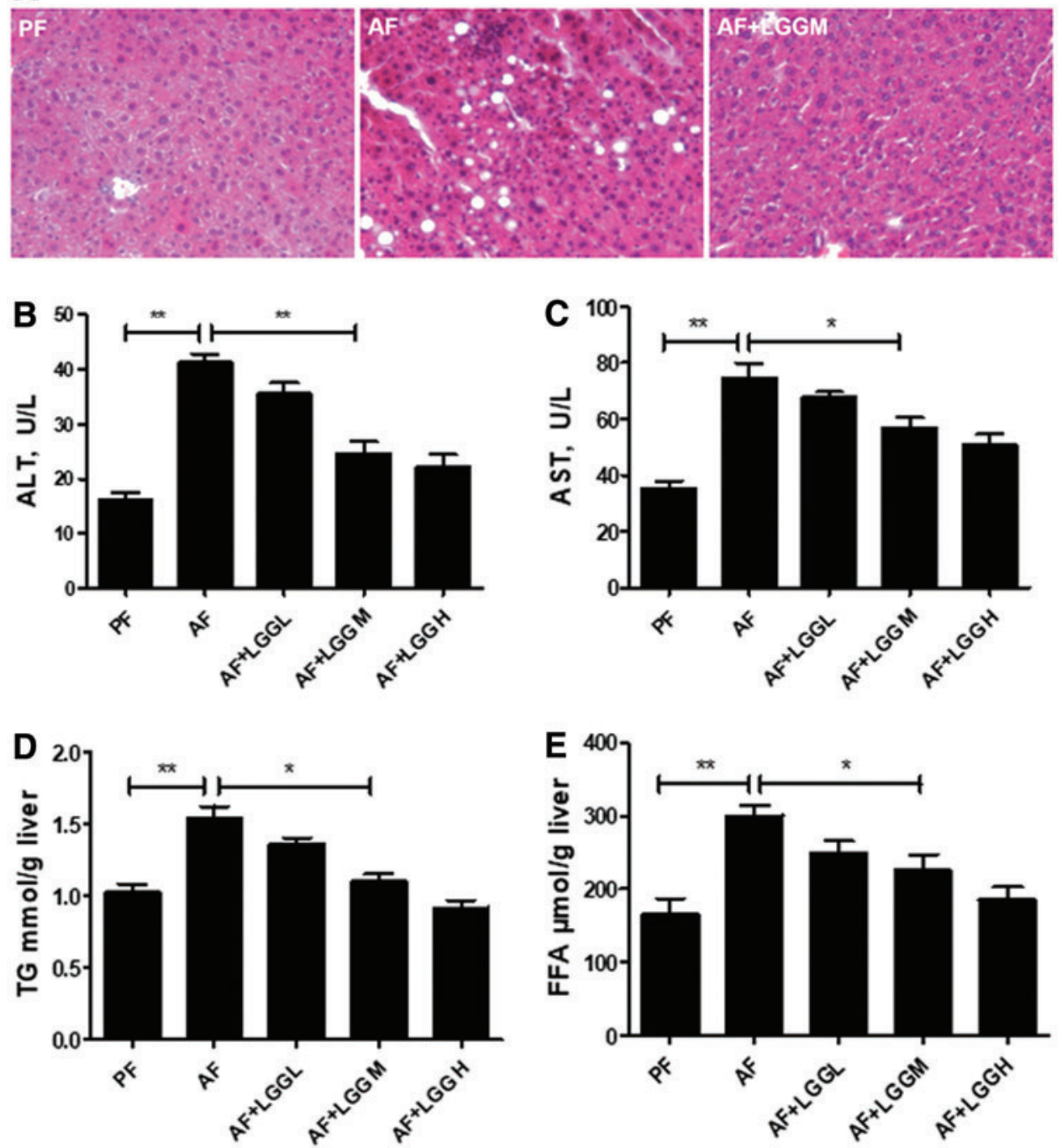

FIG. 1. Effect of LGG granule supplementation on chronic alcoholinduced liver steatosis. Mice were fed alcohol; or isocaloric maltose dextrin for 8 weeks; LGG granules were fed during the last 2 weeks. (A) Liver sections of mice were processed for staining with $\mathrm{H} \& \mathrm{E}$. Serum was obtained by centrifuging the blood at $3000 \mathrm{~g}$ for $10 \mathrm{~min}$ at $4^{\circ} \mathrm{C}$. (B) Serum levels of ALT and (C) AST were measured by using Assay kits. (D) Liver TG level and (E) FFA level were measured using kits. Values are presented as mean \pm SEM $(n=10) . * P<.05$ and $* * P<.01$ indicate the significant differences between different groups. AF, alcohol-fed; ALT, alanine aminotransferase; AST, aspartate aminotransferase; FFA, free fatty acid; H\&E, hematoxylin and eosin; LGG, Lactobacillus rhamnosus GG; LGGH, LGG high; LGGL, LGG low; LGGM, LGG middle; PF, pair-fed; SEM, standard error of the mean; TG, triglyceride. Color images are available online. 
in the AF group was higher than the PF group (Fig. 1D), which indicated liver steatosis increased by alcohol exposure $(P<.01)$. LGG granules supplementation abolished alcohol-induced TG level increase. In addition, the level of FFA was increased in the AF group (Fig. 1E), which indicated the body oxidative stress increased $(P<.01)$. LGG granule treatment blocked the alcohol-induced FFA increase. Especially, these attenuating trends were more obvious in the high dose LGG granule $(\mathrm{AF}+\mathrm{LGGH})$ group and returned to normal level. Taken together, LGG granules dose-dependently attenuated alcohol-induced liver steatosis.

\section{Effect of LGG granules supplementation on chronic alcohol-induced inflammation}

To assess hepatic inflammation, levels of serum LPS and TNF- $\alpha$ were measured. LPS is a component of Gramnegative bacteria cell walls and an important pathogenic substance that causes oxidative stress and inflammatory responses. ${ }^{13}$ Compared with the PF group, alcohol-induced serum LPS significantly increased (Fig. 2A) $(P<.01)$. LGG granule supplements abolished the alcohol-induced LPS level increase. In AF group the level of TNF- $\alpha$ was higher than PF group (Fig. 2B), and it was remarkably decreased in $\mathrm{AF}+\mathrm{LGGM}$ group $(P<.01)$. Likewise, LGG granule supplements had a significant effect on decreasing production of inflammatory cytokines.

\section{Effect of $L G G$ granules supplementation on chronic alcohol-induced hepatic MDA activation}

Alcohol-exposure-mediated lipid peroxidation is an important cause of liver injury. MDA is a byproduct of lipid peroxidation and the amount of MDA indirectly reflects the extent of liver damage. ${ }^{27} \mathrm{We}$ measured the level of MDA in liver and found hepatic MDA levels in the AF group were higher than in the PF group $(P<.01)$, which indicated liver lipid peroxidation induced by alcohol exposure (Fig. 3). LGG granule supplementation suppressed alcohol-induced production of MDA, and the inhibitory effect of LGG granule on MDA was most significant in the $\mathrm{AF}+\mathrm{LGGH}$ group.
Effect of LGG granule supplementation on the changes of ileum Lactobacillus, Bifidobacterium, and $\mathrm{C}$. perfringens in chronic alcohol model

Research had found that alcohol consumption can cause overgrowth of intestinal Gram-negative bacteria, resulting in intestinal flora imbalance. ${ }^{28}$ Many clinical trials showed that chronic alcohol leads to an imbalance of intestinal flora that is characterized by the decrease of Lactobacillus and Bifidobacterium. ${ }^{29}$ In this study, we collected ileum samples, and Lactobacillus, Bifidobacterium, and C. perfringens in ileum content were cultured as far as possible. Compared with the PF group, the amount of Lactobacillus (Fig. 4A) and Bifidobacterium (Fig. 4B) in the AF group was significantly decreased and that was obviously increased in the $\mathrm{AF}+\mathrm{LGGM}$ group $(P<.01)$, especially in the AF + LGGH group; whereas, compared with the PF group, the amount of $C$. perfringens in the $\mathrm{AF}$ group was significantly increased (Fig. 4C) $(P<.05)$, obviously reduced in the AF+LGGM group, especially in AF+LGGH group. The results indicated LGG granule supplementation restores alcoholinduced changes of flora in the ileum.

\section{Effect of LGG granule supplementation on cecum microflora diversity in chronic alcohol-induced mice}

Compared with the traditional microbial culture, the microgram sequencing can be found in the environment, which is difficult to develop in noncultivable microorganisms. Metagenomics can reveal micro-ecological prehidden species diversity. In our previous studies, we used metagenomic analyses of alcohol-induced alterations of the fecal microbiome via high-throughput sequencing and bioinformatics analysis, ${ }^{30,31}$ and we found that alcohol intake can lead to decline in Bacteroides and Firmicutes and increase in Proteobacteria and Actinobacteria in the mice feces. ${ }^{14}$ In the present study, we found that alcohol intake can promote the growth of more pathogenic Gram-negative bacteria, such as Proteobacteria, Campylobacter, Helicobacter, and others in mice cecum, whereas LGG granule administration inhibited alcohol-induced intestinal microbiome dysfunction via reducing the number of Gram-negative bacteria.
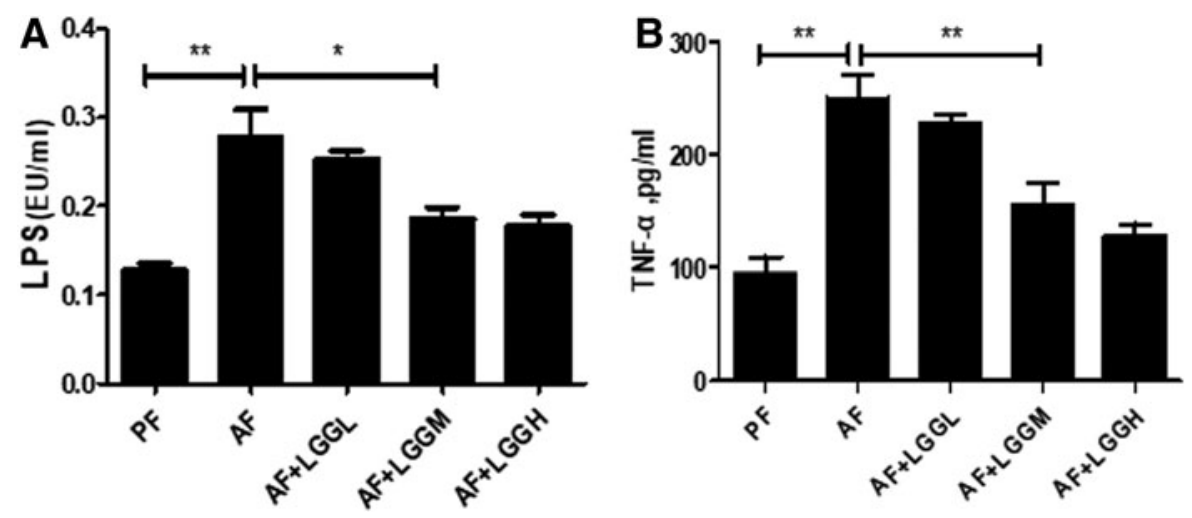

FIG. 2. Effect of LGG granule supplementation on chronic alcohol-induced inflammation. (A) Serum LPS level and (B) liver TNF- $\alpha$ level were measured by using kits. Values are presented as mean $\pm \operatorname{SEM}(n=10)$. $* P<.05$ and $* * P<.01$ indicate the significant differences between different groups. LPS, lipopolysaccharide; TNF- $\alpha$, tumor necrosis factor- $\alpha$. 


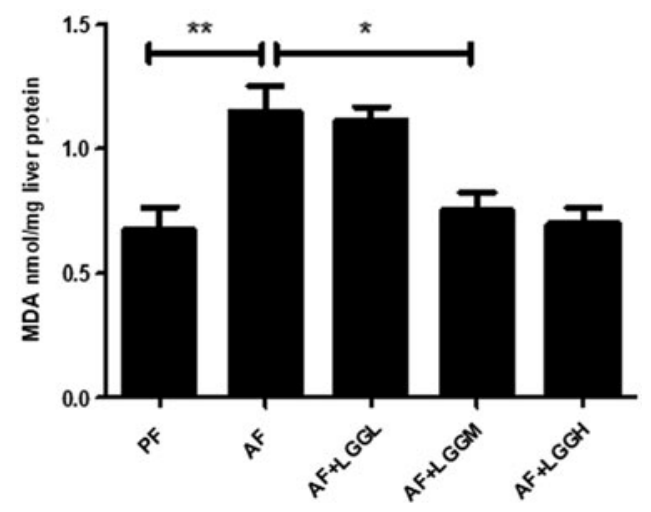

FIG. 3. Effect of LGG granule supplementation on chronic alcoholinduced hepatic MDA activity. For liver MDA assay, $\sim 100 \mathrm{mg}$ of liver tissue was homogenized in $0.9 \mathrm{~mL}$ of $0.9 \% \mathrm{NaCl}$. Liver supernatant was obtained by centrifuging the homogenate at $1800 \mathrm{~g}$ for $10 \mathrm{~min}$ at $4^{\circ} \mathrm{C}$. MDA level was measured using kit. Values are presented as mean $\pm \operatorname{SEM}(n=10) . * P<.05$ and $* * P<.01$ indicate the significant differences between different groups. MDA, malondialdehyde.

\section{Effect of LGG granule supplementation on the bacterial diversity changes at the level of phylum}

The abundance of cecum bacteria phyla levels was analyzed by metagenomics (Fig. 5), which showed that the number of Firmicutes, Bacteroidetes, and Proteobacteria were the most prominent phyla in cecum microflora, and
Firmicutes was significantly decreased and Proteobacteria was increased in the AF group. However, the number of Firmicutes was elevated and Proteobacteria was decreased in $\mathrm{AF}+\mathrm{LGG}$ group. This demonstrated that LGG could alleviate reductions in Firmicutes and expansion of Proteobacteria.

\section{Effect of LGG granules supplementation on the bacterial diversity changes at the order level}

The relative abundance of cecum bacteria was analyzed at the order level (Fig. 6A, B). In comparison to PF group, the AF group showed the greatest shifts, and revealed reductions in both Clostridiales and Lactobacillales, and an increase in Campylobacteralse. Importantly, LGG treatment was significantly able to increase the number of Clostridiales and Lactobacillales and decrease the number of Campylobacterales.

\section{Effect of LGG granule supplementation} on the bacterial diversity changes at the genus level

Relative abundance of the dominant bacterium in cecum was detected at the genus level and is shown in Figure 7A. Each bar represents the relative abundance of each sample. Also, the microbiome of the PF (right), AF+LGG (left), and AF (middle lower) groups are shown in the pie charts (Fig. 7B) and the different shades of color represent the
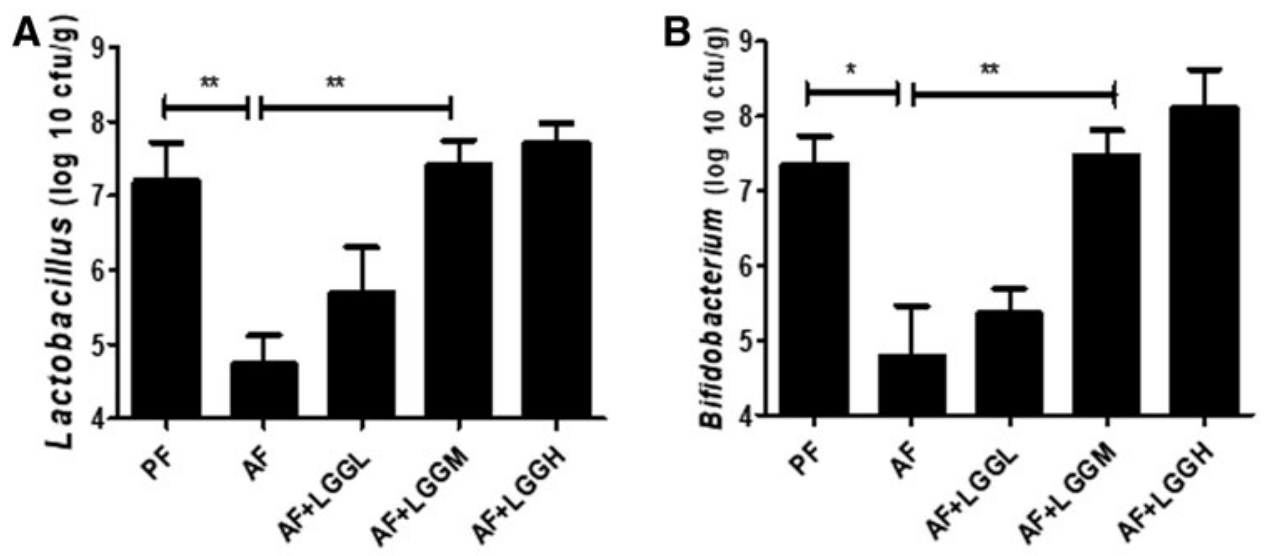

FIG. 4. Effects of LGG granule supplementation on chronic alcoholinduced changes in flora in the ileum. The feces of mice were collected after alcohol administration and LGG granules treatment for 8 weeks, feces samples were maintained as far as possible using the traditional culture, and then tested for the number of

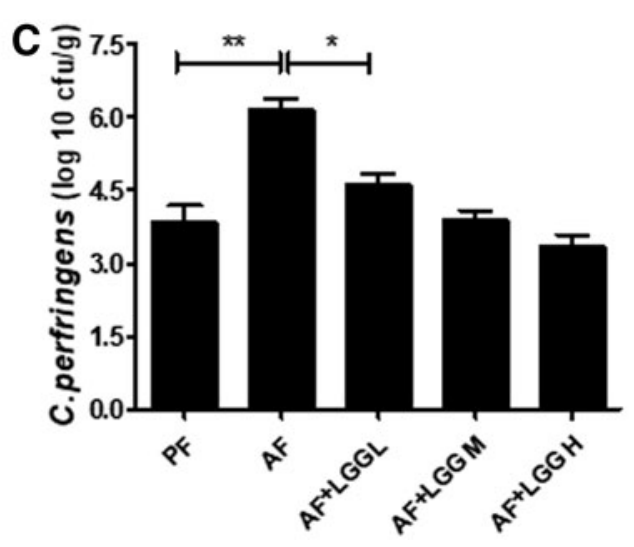
(A) Lactobacillus, (B) Bifidobacterium, and (C) Clostridium perfringens, analysis of LGG granules treatment on chronic alcohol-induced changes in the number of ileum flora. Values are presented as mean \pm SEM $(n=10)$. $* P<.05$ and $* * P<.01$ indicate significant differences between different groups. 


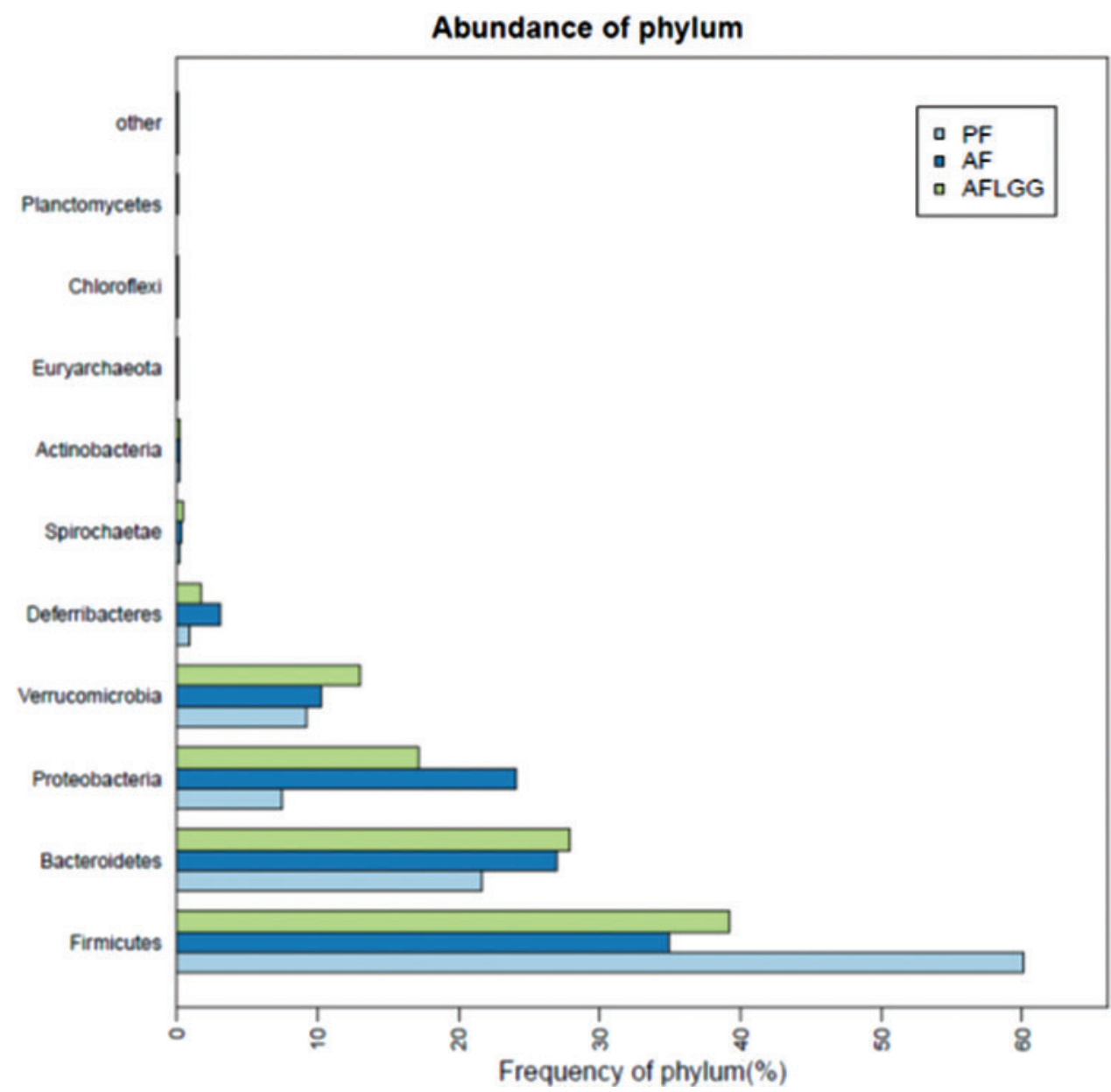

FIG. 5. Relative abundance of the dominant cecum bacterial phylum in cecum of chronic alcohol-induced mice. Color images are available online. different genera. The cecum microbiomes of mice exposed to alcohol were characterized by greater abundance of $\mathrm{He}$ licobacter and loss of unclassified-Ruminococcaceae. In the $\mathrm{AF}+\mathrm{LGG}$ group, the abundance of unclassified-Ruminococcaceae and Lactobacillus were increased, and Helicobacter was significantly decreased, compared with the AF group.

\section{DISCUSSION}

So far, the exact pathogenesis of ALD is not clear. It is believed that long-term and excessive alcohol intake changes intestinal microbial diversity, reduces the intestinal mucus layer and epithelial tight junction protein expression leading to intestine barrier dysfunction, and endotoxemia and lipid peroxidation in liver is enhanced and induce a large number of free radicals that cause oxidative damage of liver tissue. ${ }^{27}$ Recently, probiotics have been used as a kind of functional microbes, these have good physiological and biochemical functions for humans, and some researchers have demonstrated that probiotics can effectively attenuate alcoholic liver injury. ${ }^{32-35}$ Forsyth et al., showed the correlation of ALD with oxidative stress and pointed out that LGG could maintain intestinal barrier function by reducing alcohol-oxidative stress and improve the alcoholic fatty liver disease. ${ }^{36}$ In previous studies, we demonstrated that
LGG could improve alcohol-induced intestinal barrier dysfunction and decrease endotoxemia in blood via increasing intestinal tight junction proteins and mucous protein levels, ${ }^{13}$ which can ameliorate alcohol-induced liver injury. LGG-s prevented acute alcoholic intestinal barrier dysfunction and bacterial endotoxemia and protect the gut integrity and permeability. ${ }^{22}$ LGG-s treatment decreased alcohol-induced inflammatory cytokine $(\mathrm{TNF}-\alpha)$ production via inhibition of Toll-like receptor (TLR)4- and TLR5mediated endotoxin activation. ${ }^{15}$

It is difficult to store and transport LGG cells and LGG-s, and the durability period is about 15 days at freezing temperatures. LGG granules prepared by fluidized bed granulator, which were composed of starch, skimmed milk powder, whey powder, microcrystalline cellulose and maltose, and LGG fermented liquid were $30-50 \%$ of the total weight, can be stored at $0-4^{\circ} \mathrm{C}$ for 6 months. The results of this study suggested that the dose dependence of LGG granules was effective for protecting against chronic alcoholinduced liver injury and regulating gut microbiota.

There is a close relationship between gut microbiota and human health, they play an important role in the pathogenesis of liver disease. ${ }^{13,37,38}$ Both chronic and acute alcohol consumption can lead to intestinal dysbiosis and pathogenic bacterial overgrowth ${ }^{39-43}$ that promote intestinal chronic inflammation. ${ }^{44,45}$ A previous study showed probiotics 



FIG. 6. Effects of LGG treatment on cecum bacterial diversity changes at the order level. (A) The relative abundance of bacterial order was indicated by group merge. Each bar represents the relative abundance of each group. Each color represents a particular bacterial order. (B) Heat map of the abundance of chronic alcohol-induced mice cecum bacteria at the order level. Phylogenetic positions are projected by the OTUs, and the taxa of OTUs are listed on the right. Color intensity indicates the relative enrichment of OTUs. OTUs, operational taxonomic units. Color images are available online.

Desulfovibrionales

Lactobacillales

Burkholderiales

Deforribacterales

Enterobacteriales

Spirochaetales

Methanosarcinales

Pseudomonadales

Rhodospirillales

Selenomonadales

\&

$\$$

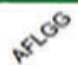

0.35
0.6
8.83 
A

A 100

Distribution Barplot

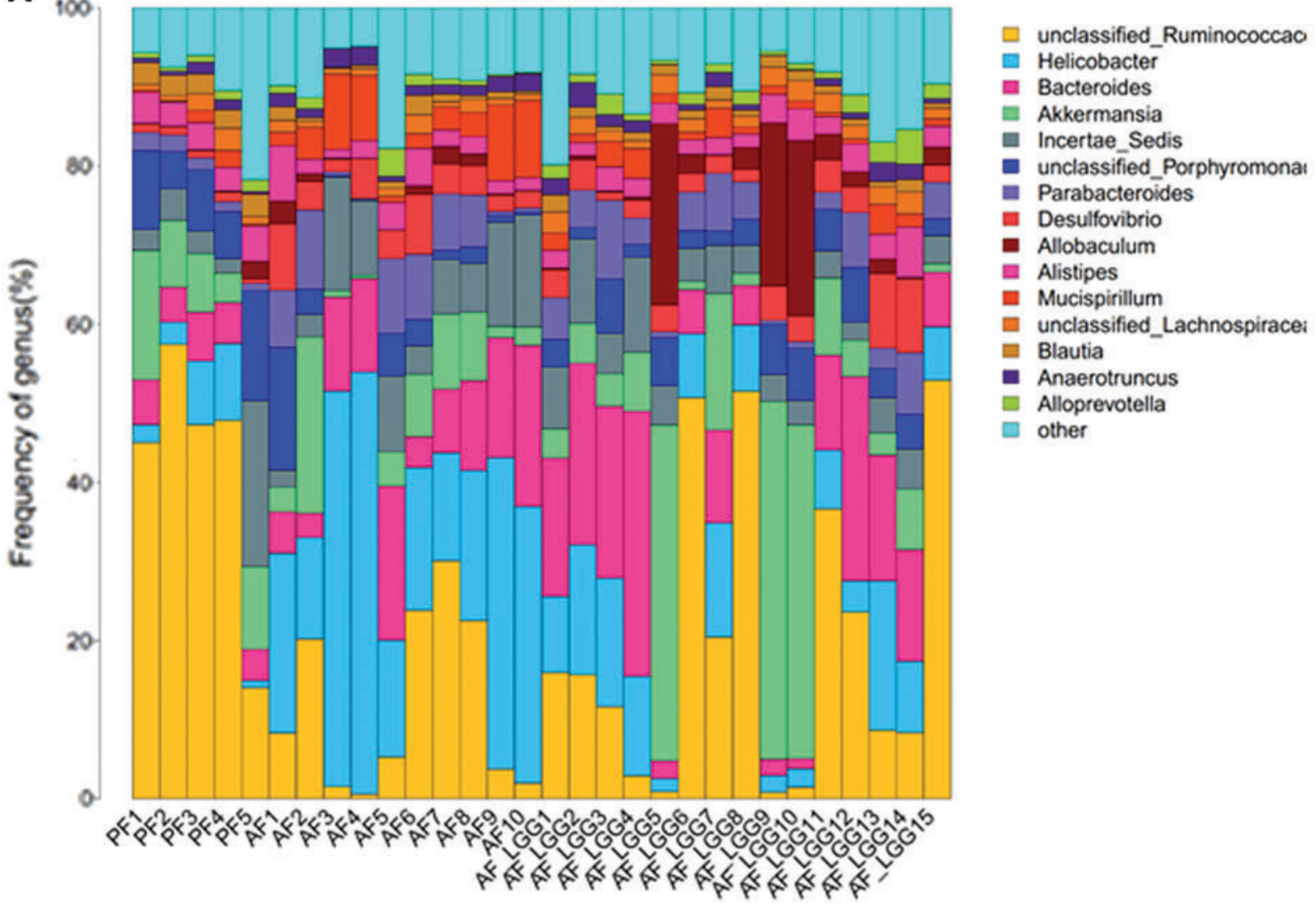

B

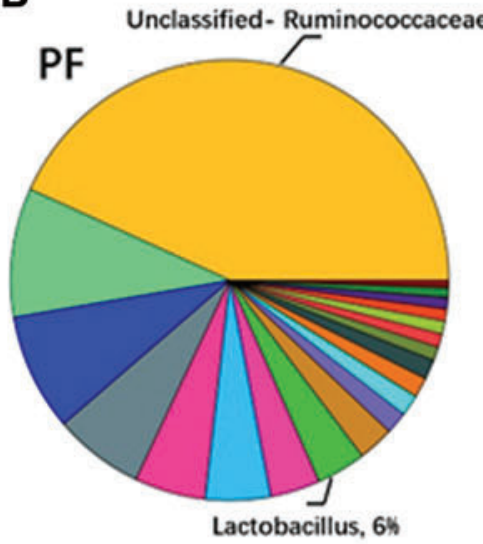

$A F+L G G$

Helicobacter, 19\%

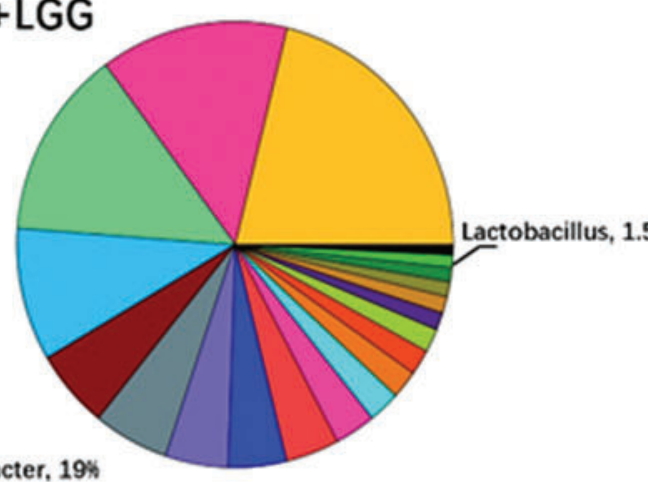

ㅁ unclassified_Ruminococcaceae

Bacteroides

ㅁ Akkermansia

口 Helicobacter

- Allobaculum

口 Incertae_Sedis

口 Parabacteroides

- unclassified_Porphyromonadaceae

口 Desulfovibrio

口 Alstipes

other

u unclassified_Lachnospiraceae

- Mucispirillum

口 Alloprevotella

- Anaerotruncus

ㅁ Blautia

Escherichia-Shigella

AF

- Variovorax

- Lactobacillus

- Parasutterella

FIG. 7. Effect of LGG treatment on cecum bacterial diversity changes at the genus level. (A) Relative abundance of the dominant bacterial in cecum at the genus level. Each bar represents the relative abundance of each sample. Each color represents a particular bacterial genus. (B) The relative distribution of bacterial genera in response to alcohol feeding and LGG granules supplementation are shown in the pie chart. Color images are available online. 
supplement increased Lactobacillus and Bifidobacterium in the patient's feces. ${ }^{6}$ This study we found that chronic alcohol exposure decreased the number of Lactobacillus and Bifidobacterium and increased the number of $C$. perfringens in mice ileum, while that was positively regulated after LGG granules treatment. Lactobacillus and Bifidobacterium can prevent the growth of anaerobic Gram-positive bacteria, inhibit the growth of Gram-negative bacteria, enhance cell phagocytic activity, and promote the secretion of $\operatorname{IgA}$, and thereby enhance the cellular immune function. ${ }^{46,47} \mathrm{C}$. perfringens is a common pathogen that can produce a lot of endotoxin in human and animal intestines, leading to endotoxemia and necrotizing enteritis.

Many researchers believe that the main cause of liver injury is that intestinal bacteria endotoxins travel through the portal vein into the liver and active Kupffer cells and cause inflammatory cytokine production. ${ }^{48}$ Under normal conditions, a small amount of intestinal bacteria endotoxin from the hepatic portal vein to the liver can be cleared by liver macrophages (Kupffer cells). ${ }^{49,50}$ We demonstrated that alcohol caused intestinal flora disruption, the number of Firmicutes, Clostridiales, and unclassified-Ruminococcaceae decreased, and Gram-negative Proteobacteria, Campylobacterales, and Helicobacter increased. These Gramnegative bacteria proliferated in the intestine and a large amount of endotoxin was produced and released into blood, which was beyond the clearance of the liver. ${ }^{13}$ The serum endotoxin (serum LPS) level in ALD animal is significantly higher than normal, the higher the endotoxin level, the more severe liver disease. ${ }^{51-54}$

In addition, the alcohol-induced Kupffer cells releases large amounts of hydroxyl radical, superoxide anion, and inflammatory cytokines such as TNF- $\alpha$, interleukin (IL)-6, and IL- $1 \beta,{ }^{55,56}$ which can damage normal hepatic cells and induce inflammation, leading to liver injury. LGG granules administration could decrease the number of $C$. perfringens and increase the Lactobacillus and Bifidobacterium in the intestine of alcohol-induced mice, which suggested LGG granules prevent alcohol-induced intestinal flora disorder, increase Gram-positive bacteria, and decrease Gramnegative bacteria that induce LPS accumulation, and reduce fat accumulation and inflammatory response in liver, and then ameliorate the liver damage.

With the deepening research of the development mechanism of ALD, it has been found that the liver produces large amounts of reactive oxygen species (ROS, such as $\mathrm{O}^{2-}, \mathrm{O}_{2}^{-}$, $\mathrm{OH}^{-}$) when it metabolizes alcohol and ROS can increase lipid peroxidation and lead to upregulated level of MDA. Likewise, chronic alcohol exposure-mediated fatty acid $\beta$-oxidation decrease in liver and cause fat liver. ${ }^{57,58}$ Previous studies have showed LGG and LGG-s treatment relieved alcohol-induced ROS accumulation in ileum ${ }^{13}$ and liver. ${ }^{22}$ Zhang found LGG-s can prevent alcohol-mediated hepatic fat accumulation via reducing the expression of sterol regulatory element-binding protein-1 (SREBP-1) and upregulating the level of Carnitine palmitoyltransferase-1 (CPT-1) and Peroxisome proliferator activated receptor- $\alpha$ (PPAR- $\alpha$ ), leading to increased fatty acid $\beta$-oxidation. ${ }^{32} \mathrm{In}$ this study we show that LGG granules prevent chronic alcohol-mediated hepatic MDA activation and attenuate lipid accumulation and inflammation in liver.

In the present study, our findings demonstrate that $0.1 \mathrm{~g} /$ (day·mouse) LGG granules can prevent alcohol-induced expansion of Gram-negative bacteria in ileum and cecum and decrease enterogenous endotoxin and inflammatory response, and then ameliorated the liver injury. The results of the study highlight the need for future research to use a more representative clinical sample.

\section{AUTHOR DISCLOSURE STATEMENT}

The authors declare that they have no conflicts of interest (financial or otherwise) related to the data presented in this article.

\section{FUNDING INFORMATION}

This work was supported by National Key R\&D Program of China (Grant No. 2017YFE0105400).

\section{REFERENCES}

1. Beier JI, Arteel GE, McClain CJ: Advances in alcoholic liver disease. Curr Gastroenterol Rep 2011;13:56-64.

2. Gao B, Bataller R: Alcoholic liver disease: Pathogenesis and new therapeutic targets. Gastroenterology 2011;141:1572-1585.

3. O'shea RS, Dasarathy S, McCullough AJ: Alcoholic liver disease. Hepatology 2010;51:307-328.

4. Frazier TH, Stocker AM, Kershner NA, Marsano LS, McClain CJ: Treatment of alcoholic liver disease. Therap Adv Gastroenterol 2011;4:63-81.

5. Hill C, Guarner F, Reid G, et al:: The International Scientific Association for Probiotics and Prebiotics consensus statement on the scope and appropriate use of the term probiotic. Nat Rev Gastroenterol Hepatol 2014;11:506-514.

6. Kirpich IA, Solovieva NV, Leikhter SN, et al.: Probiotics restore bowel flora and improve liver enzymes in human alcoholinduced liver injury: A pilot study. Alcohol 2008;42:675-682.

7. Bubnov RV, Babenko LP, Lazarenko LM, et al.: Comparative study of probiotic effects of Lactobacillus and Bifidobacteria strains on cholesterol levels, liver morphology and the gut microbiota in obese mice. EPMA J 2017;8:357-376.

8. Lata J, Jurankova J, Kopacova M, Vitek P: Probiotics in hepatology. World J Gastroenterol 2011;17:2890.

9. Natividad JM, Agus A, Planchais J, et al:: Impaired Aryl hydrocarbon receptor ligand production by the gut microbiota is a key factor in metabolic syndrome. Cell Metab 2018;28:737.e734749.e734.

10. Galdeano CM, Cazorla SI, Dumit JML, Vélez E, Perdigón G: Beneficial effects of probiotic consumption on the immune system. Ann Nutr Metab 2019;74:115-124.

11. Bubnov RV, Spivak MY, Lazarenko LM, Bomba A, Boyko NV: Probiotics and immunity: Provisional role for personalized diets and disease prevention. EPMA $J$ 2015;6:14.

12. Bubnov RV, Babenko LP, Lazarenko LM, Mokrozub VV, Spivak MY: Specific properties of probiotic strains: Relevance and benefits for the host. EPMA $J$ 2018;9:205-223.

13. Wang Y, Kirpich I, Liu Y, et al.: Lactobacillus rhamnosus GG treatment potentiates intestinal hypoxia-inducible factor, 
promotes intestinal integrity and ameliorates alcohol-induced liver injury. Am J Pathol 2011;179:2866-2875.

14. Bull-Otterson L, Feng W, Kirpich I, et al:: Metagenomic analyses of alcohol induced pathogenic alterations in the intestinal microbiome and the effect of Lactobacillus rhamnosus GG treatment. PLoS One 2013;8:e53028.

15. Wang Y, Liu Y, Kirpich I, et al.: Lactobacillus rhamnosus GG reduces hepatic $\mathrm{TNF} \alpha$ production and inflammation in chronic alcohol-induced liver injury. J Nutr Biochem 2013;24:1609_ 1615.

16. Hong M, Kim SW, Han SH, et al:: Probiotics (Lactobacillus rhamnosus R0011 and acidophilus R0052) reduce the expression of Toll-like receptor 4 in mice with alcoholic liver disease. PLoS One 2015;10:e0117451.

17. Marotta F, Barreto R, Wu C, et al.: Experimental acute alcohol pancreatitis-related liver damage and endotoxemia: Synbiotics but not metronidazole have a protective effect. Chin J Dig Dis 2005;6:193-197.

18. Chang B, Sang L, Wang Y, Tong J, Zhang D, Wang B: The protective effect of VSL\#3 on intestinal permeability in a rat model of alcoholic intestinal injury. BMC Gastroenterol 2013;13:151.

19. Dhiman RK, Rana B, Agrawal S, et al.: Probiotic VSL\# 3 reduces liver disease severity and hospitalization in patients with cirrhosis: A randomized, controlled trial. Gastroenterology 2014; 147:1327.e1323-1337.e1323.

20. Stadlbauer V, Mookerjee RP, Hodges S, Wright GA, Davies NA, Jalan R: Effect of probiotic treatment on deranged neutrophil function and cytokine responses in patients with compensated alcoholic cirrhosis. J Hepatol 2008;48:945-951.

21. Segawa S, Wakita Y, Hirata H, Watari J: Oral administration of heat-killed Lactobacillus brevis SBC8803 ameliorates alcoholic liver disease in ethanol-containing diet-fed C57BL/6N mice. Int J Food Microbiol 2008;128:371-377.

22. Wang Y, Liu Y, Sidhu A, Ma Z, McClain C, Feng W: Lactobacillus rhamnosus GG culture supernatant ameliorates acute alcohol-induced intestinal permeability and liver injury. Am J Physiol Gastrointest Liver Physiol 2012;303:G32-G41.

23. Li P, Niu Q, Wei Q, et al.: Microbial shifts in the porcine distal gut in response to diets supplemented with Enterococcus faecalis as alternatives to antibiotics. Sci Rep 2017;7:41395.

24. Maidak BL, Olsen GJ, Larsen N, Overbeek R, McCaughey MJ, Woese CR: The RDP (Ribosomal Database Project). Nucleic Acids Res 1997;25:109-110.

25. Schloss PD, Westcott SL, Ryabin T, et al.: Introducing mothur: Open-source, platform-independent, community-supported software for describing and comparing microbial communities. Appl Environ Microbiol 2009;75:7537-7541.

26. Kuczynski J, Stombaugh J, Walters WA, González A, Caporaso JG, Knight R: Using QIIME to analyze 16S rRNA gene sequences from microbial communities. Curr Protoc Microbiol 2012; chapter 10:1E.5.1-1E.5.20.

27. Dey A, Cederbaum AI: Alcohol and oxidative liver injury. $\mathrm{He}$ patology 2006;43(Suppl. 1):S63-S74.

28. Nanji AA, Khettry U, Sadrzadeh S, Yamanaka T: Severity of liver injury in experimental alcoholic liver disease. Correlation with plasma endotoxin, prostaglandin E2, leukotriene B4, and thromboxane B2. Am J Pathol 1993;142:367.

29. Inokuchi S, Tsukamoto H, Park E, Liu ZX, Brenner DA, Seki E: Toll-like receptor 4 mediates alcohol-induced steatohepatitis through bone marrow-derived and endogenous liver cells in mice. Alcohol Clin Exp Res 2011;35:1509-1518.

30. Pascual M, Castilla-Cortazar I, Urdaneta E, et al.: Altered intestinal transport of amino acids in cirrhotic rats: The effect of insulin-like growth factor-I. Am J Physiol Gastrointest Liver Physiol 2000;279:G319-G324.

31. Wu C, Li M, Chen C: Endotoxin-induced liver injury and plasma tumor necrosis factor alfa, interleukin 6 level changes in rabbits. Chin J Dig 1995;15:256-258.

32. Zhang M, Wang C, Wang C, et al.: Enhanced AMPK phosphorylation contributes to the beneficial effects of Lactobacillus rhamnosus GG supernatant on chronic-alcohol-induced fatty liver disease. J Nutr Biochem 2015;26:337-344.

33. Markowiak P, Śliżewska K: Effects of probiotics, prebiotics, and synbiotics on human health. Nutrients 2017;9:1021.

34. Patel D, Patel F, Mandal P: Potential molecular mechanism of probiotics in alcoholic liver disease. J Alcohol Drug Depend 2017;5:2.

35. Shao T, Zhao C, Li F, et al:: Intestinal HIF- $1 \alpha$ deletion exacerbates alcoholic liver disease through inducing intestinal dysbiosis and barrier dysfunction. J Hepatol 2018;69:886-895.

36. Forsyth CB, Farhadi A, Jakate SM, Tang Y, Shaikh M, Keshavarzian A: Lactobacillus GG treatment ameliorates alcoholinduced intestinal oxidative stress, gut leakiness, and liver injury in a rat model of alcoholic steatohepatitis. Alcohol 2009;43:163172.

37. Cesaro C, Tiso A, Del Prete A, et al.: Gut microbiota and probiotics in chronic liver diseases. Dig Liver Dis 2011;43: 431-438.

38. Loguercio C, Federico A, Tuccillo C, et al.: Beneficial effects of a probiotic VSL\# 3 on parameters of liver dysfunction in chronic liver diseases. J Clin Gastroenterol 2005;39:540-543.

39. Albano E: Oxidative mechanisms in the pathogenesis of alcoholic liver disease. Mol Aspects Med 2008;29:9-16.

40. McLoughlin RM, Mills KH: Influence of gastrointestinal commensal bacteria on the immune responses that mediate allergy and asthma. J Allergy Clin Immunol 2011;127:1097-1107.

41. Canesso MCC, Lacerda N, Ferreira C, et al.: Comparing the effects of acute alcohol consumption in germ-free and conventional mice: The role of the gut microbiota. BMC Microbiol 2014;14:240.

42. Bajaj JS, Heuman DM, Hylemon PB, et al.: Altered profile of human gut microbiome is associated with cirrhosis and its complications. J Hepatol 2014;60:940-947.

43. Gabbard SL, Lacy BE, Levine GM, Crowell MD: The impact of alcohol consumption and cholecystectomy on small intestinal bacterial overgrowth. Dig Dis Sci 2014;59:638-644.

44. Bajaj JS, Hylemon PB, Ridlon JM, et al:: Colonic mucosal microbiome differs from stool microbiome in cirrhosis and hepatic encephalopathy and is linked to cognition and inflammation. Am J Physiol Gastrointest Liver Physiol 2012;303: G675-G685.

45. Mutlu EA, Gillevet PM, Rangwala H, et al:: Colonic microbiome is altered in alcoholism. Am J Physiol Gastrointest Liver Physiol 2012;302:G966-G978.

46. Zhang W, Gu Y, Chen Y, et al.: Intestinal flora imbalance results in altered bacterial translocation and liver function in rats with experimental cirrhosis. Eur J Gastroenterol Hepatol 2010;22: 1481-1486. 
47. Wang Y, Liu L, Moore DJ, et al:: An LGG-derived protein promotes IgA production through upregulation of APRIL expression in intestinal epithelial cells. Mucosal Immunol 2017;10: 373.

48. Thurman R: II. Alcoholic liver injury involves activation of Kupffer cells by endotoxin. Am J Physiol Gastrointest Liver Physiol 1998;275:G605-G611.

49. Rao R, Seth A, Sheth P: Recent advances in alcoholic liver disease I. Role of intestinal permeability and endotoxemia in alcoholic liver disease. Am J Physiol Gastrointest Liver Physiol 2004;286:G881-G884.

50. Wheeler MD: Endotoxin and Kupffer cell activation in alcoholic liver disease. Alcohol Res Health 2003;27:300-306.

51. Fujimoto M, Uemura M, Nakatani Y, et al.: Plasma endotoxin and serum cytokine levels in patients with alcoholic hepatitis: Relation to severity of liver disturbance. Alcohol Clin Exp Res 2000;24(4 Suppl.):48S-54S.

52. Hanck C, Rossol S, Böcker U, Tokus M, Singer MV: Presence of plasma endotoxin is correlated with tumour necrosis factor receptor levels and disease activity in alcoholic cirrhosis. Alcohol Alcohol 1998;33:606-608.
53. Mathurin P, Deng QG, Keshavarzian A, Choudhary S, Holmes EW, Tsukamoto H: Exacerbation of alcoholic liver injury by enteral endotoxin in rats. Hepatology 2000;32:1008-1017.

54. Nanji AA, Jokelainen K, Fotouhinia M, et al.: Increased severity of alcoholic liver injury in female rats: Role of oxidative stress, endotoxin, and chemokines. Am J Physiol Gastrointest Liver Physiol 2001;281:G1348-G1356.

55. Fukui H: Relation of endotoxin, endotoxin binding proteins and macrophages to severe alcoholic liver injury and multiple organ failure. Alcohol Clin Exp Res 2005;29(Suppl. 2):172S-179S.

56. Hines IN, Wheeler MD: Recent advances in alcoholic liver disease III. Role of the innate immune response in alcoholic hepatitis. Am J Physiol Gastrointest Liver Physiol 2004;287: G310-G314.

57. García-Villafranca J, Guillén A, Castro J: Ethanol consumption impairs regulation of fatty acid metabolism by decreasing the activity of AMP-activated protein kinase in rat liver. Biochimie 2008;90:460-466.

58. You M, Liang X, Ajmo JM, Ness GC: Involvement of mammalian sirtuin 1 in the action of ethanol in the liver. Am J Physiol Gastrointest Liver Physiol 2008;294:G892-G898. 